\title{
A Novel Immunological Technique for Detection of Gramicidin (S) Producing Bacteria
}

\author{
Fouad Houssein Kamel \\ Erbil Medical Technical Institute, Erbil, Iraq \\ Email: fhkamel2005@yahoo.com
}

Received December 21, 2011; revised January 17, 2012; accepted April 22, 2012

\begin{abstract}
A serological kit was prepared for the first time to detect bacteria that produce Gramicidin (S). Since, an immunocomplex of antibiotic Gramicidin (S) was prepared followed by immunonization of laboratory animales to obtain specific antibodies in their serum. Serial dilutions of the prepared antibodies were tested directly against samples of bacteria and compared with control samples, which include bacteria that produce Gramicidin (S) (as a positive control) and the bacteria that does not produce Gramicidin (S) (as a negative control) depending slide method after the incubation of bacteria in a suitable media for $3-4$ hours at $37^{\circ} \mathrm{C}$. The prepared kites were evaluated in reference Laboratories and they proved to be highly sensitive (100\%), specific $(100 \%)$, more economic and reliable procedure, in addition to its stability for more than one year. It was the first time to prepare a kit for detection of bacteria which produce Gramicidin (S) directly within few minutes.
\end{abstract}

Keywords: Gramicidin; Serological Kit; Immuno-complex

\section{Introduction}

Gramicidin (S) (Scheme 1) has historically been employed as a topical antibiotic for the treatment of infections from superficial wounds. It exhibits strong antibiotic activity against a broad spectrum of Gram negative, Gram-positive bacteria and against several pathogenic fungi [1]. Gramicidin (S) produced by the Gram positive bacterium $B a$ cillus brevis, it is a cyclodecapeptide, constructed as two identical penta peptides joined head to tail, formally written as cycle (-Val-Orn-Leu-D-Phe-Pro-) $)_{2}$ [2]. It forms a ring structure composed of five different amino acids, each one used twice within the structure [3].

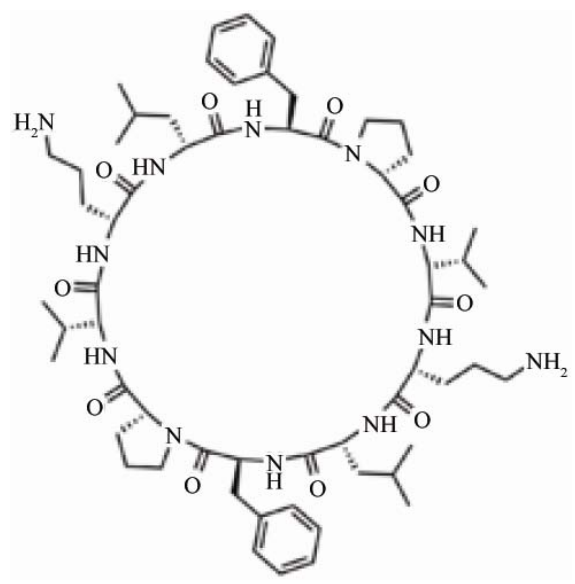

Scheme 1. Gramicidin (S).
Another interesting point is that it utilizes two amino acids uncommon in peptides, ornithine as well as the unnatural stereoisomer of phenylalanine. It is synthesized by Gramicidin (S) synthetase [4]. This antibiotic did not melt in water or weak acid and weak alkaline solution, but rapidly melt in alcohols, with melting point $\left(226^{\circ} \mathrm{C}\right.$ $\left.227^{\circ} \mathrm{C}\right)$ and molecular weight ranges between (10601340) Dalton. It's resistant to high temperatures of up to $\left(121^{\circ} \mathrm{C}\right)$ for period of $(90)$ minutes [5].

The purpose of this research was to find a rapid and accurate immunological method for detection of antibiotic Gramicidin (S) producing bacteria, by using antibiotic immunogen to produce an antibody specific to Gramicidin (S).

The study showed that out of 12 isolates of B. brevis, only four isolates had the ability to produce Gramicidin (S) antibiotic, since the isolate $B$. brevis (Bb3) was most efficient in production of the antibiotic [6]. It was suggested that strains produce Gramicidin (S) does not produce other peptide antibiotics [7]. The purpose of this research was to find a direct immunological technique characterized with sensitivity and specificity for the detection of antibiotic producing bacteria in short period.

\section{Materials \& Methods}

\subsection{Immune Complex Preparation (Binding of Gramicidin (S) with BSA)}

Added 2.1 Gram of the Bovin Serum Albomin (BSA) to 
$15 \mathrm{ml}$ distilled water without mixing.

Added $40 \mathrm{mg}$ of the Gramicidin(S) (Gra.(S)) to $5 \mathrm{ml}$ of ethanol alcohol (95\%) dissolve $30 \mathrm{mg}$ of Carbon Diamide (CDI) in $5 \mathrm{ml}$ of Di-methyl Formamide (DMF).

Later $4 \mathrm{ml}$ of solution (CDI) was added to the solution (BSA) and kept for half hour with continuous mixing.

Gramicidin (S) solution was added drop wise to solution (CDI-BSA) with continuously mixing. The mixture left for interaction (18) hours then dialyzed against distilled water for 48 hours [8].

\subsection{Test the Immune Complex}

1) Cellulose acetate paper electrophoresis: Test was done by using cellulose acetate paper electrophoresis in Educational Medical City laboratories [9].

2) Paper electrophoresis [10].

\subsection{Production of the Antibody (Anti-Garamicidine (S))}

Immunogen was prepared by mixing the immunocomplex once with complete freud adjuvant and later with incomplete freud adjuvant as a booster dose [11]. Laboratory animals were injected intramuscularly and bleeded after each booster dose to obtain serum.

Serial dilutions of serum were tested against Gramicidin (S) as antigen using precipitation ring method [12].

\subsection{Final Diagnosis Kit}

The prepared kit consists of:

1) Reagent: Included best dilution of anti-serum.

2) Positive control: Bacterial cell suspention (productive strain) in phosphate buffer.

3) Negative control: Bacterial cell suspention (non productive strain) in phosphate buffer.

\section{Results \& Discussion}

Figure 1 showed the immune complex model which was examined in comparison with both free BSA and Gramicidin (S). Results of the analysis forms three separated different bands, the BSA band had cleared the site and the status of normal, while the synthesized immune complex (BSA bond with Gramicidin (S)) at the site before with a wider area than albumin band, whatever the same concentration of both free and bond BSA had been used in the experiment and the method adopted was cellulose acetated paper electrophoresis $[6,13]$.

The free antibiotic has not shown the opposite which may be deported because of concentration, charge or a lack of focus of the article.

Figure 2 showed previous result using a paper electrophoresis. It was also stressed the state of the link through the complex nature consisting compared with both free

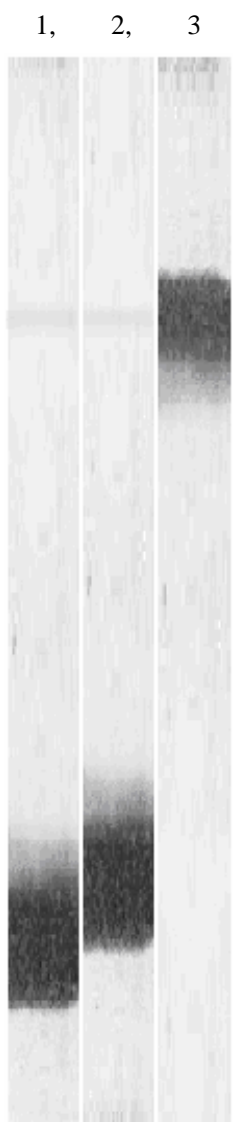

Figure 1. Cellulose acetated paper electrophoresis ran (1: ASA, 2: BSA-Gra.S, 3: Gra.S).

antibiotic and BSA. This stain was depended in our study because of its nature that engages in the installation of Gramicidin only.

Gramicidin (S) is a material of low molecular weight was not able to stimulate the immune system of the laboratory animals and production of required antibodies. Adopted many ways to prepare complexes antibiotic with the types of protein of high molecular weight such as BSA was found through experiments to produce new complex $[6,14,15]$. Thus, the status of the link has been required to prepare the appropriate antigen with high molecular weight and can stimulate the immune system $[13,16]$. The types of amino acids are help to make the link with the BSA through the effective groups including carboxyl and amines, which helps to link with a carbon diamide [15,17]. As a results of active and booster dose immunizing laboratory animals with prepared immune complex which has stimulated the immune system of the animals through production of antibody against Gramicidin (S) and that also proved the success of the research procedures. The antibody was tested against the antibiotic using precipitation ring test [18], which showed atypical precipitation ring in the case of reaction between antibiotic (as antigen) and specific antibody if its present. As a 


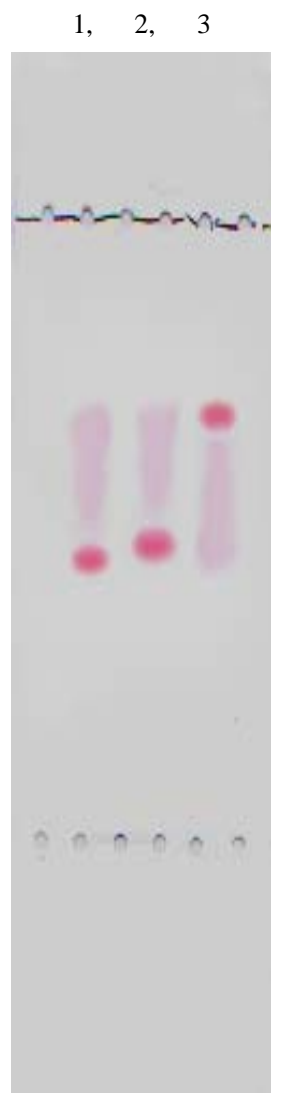

Figure 2. Paper electrophoresis ran (1: ASA, 2: BSA-Gra.S, 3: Gra.S).

result of this research we may be able to prepare Gramicidin (S) immunocomplex to obtain specific antibody, which could be depended in prepation of specific diagnostic kit. Thease methods are non expensive and thus it is standard practice to test only pre-screened individuals rather than, for example, mass-screening of patients upon admission to hospital. Apart from the (very expensive) DNA testing methods, all of the known methods require pure cultures of bacteria which have to be obtained from the individual and then grown over at least 48 hours prior to testing. This means that these known tests cannot be used to obtain rapid results which would allow infection control measures to be implemented at the earliest possible opportunity. The process could be applied to other antibiotics which have composed of amino acid.

\section{Conclusion}

This manuscript aims to find a rapid and accurate immunological method for detection of antibiotic Gramicidin (S) producing bacteria, by using antibiotic immunogen to produce an antibody specific to Gramicidin (S).

\section{REFERENCES}

[1] G. Nagamurthi and S. Rambhav, "Gramicidin (S): Struc-
ture-Activity Relationship,” Journal of Biosciences, Vol. 7, No. 3-4, 1985, pp. 323-329.

[2] W. Peter, "Three-Component Synthesis of $\alpha, \beta$-Cyclopropyl- $\gamma$-Amino Acids,” American Chemical Society, Division of Organic Chemistry, Vol. 127, No. 206, 2005, pp. 5742-5743.

[3] A. Llamas-Saiz, "Double-Stranded Helical Twisted $\beta$-Sheet Channels in Crystals of Gramicidin (S) Grown in the Presence of Trifluoroacetic and Hydrochloric Acids," Acta Crystallographica Section D, Vol. 63, No. 3, 2007, pp. 401-407. doi:10.1107/S0907444906056435

[4] P. Brick, "Structural Basis for the Activation of Phenylalanine in the Non-Ribosomal Biosynthesis of Gramicidin S," The EMBO Journal, Vol. 16, No. 14, 1997, pp. 4174-4183. doi:10.1093/emboj/16.14.4174

[5] T. Korzybaski, Z. Kowszk and W. Kurylowicz, "Antibiotics: Origin, Nature and Properties,” Pergamon Press, Oxford, London, New York, 1967.

[6] E. D. Snell and G. T. Snell, "Calorimetric Method of Analysis,” Van Nostr and Reinhold Company, Newark, Toranto, London, 1971.

[7] E. Kate and A. L. Demaine, "The Peptide Antibiotic of Bacillus: Chemistry, Biogenesis, and Possible Functions,” Bacteriology Reviews, Vol. 41, No. 2, 1977, pp. 449-474.

[8] S. S. LateEf, S. Gupta, L. P. Jayathlilaka, S. Krishnanchettia, J. S. Huanq and B. S. Lee, “An Improved Protocol for Coupling Synthetic Peptides to Carrier Proteins for Antibody Production Using DMF to Solubilize Peptides," Journal of Biomolecular Techniques, Vol. 18, No. 3, 2007, pp. 173-176.

[9] L. P. Cawley, "Electrophoresis and Immunoelectrophoresis,” Little Brown Co., Boston, 1973.

[10] W. P. Jencks, M. R. Jetton and E. L. Durrum, "Paper Electrophoresis as a Quantitative Method. Serum Proteins,” Biochemical Journal, Vol. 60, No. 2, 1955, pp. 205-215.

[11] A. Gavin, K. Hoebe, B. Duong, T. Ota, C. Martin, B. Beutler and D. Nemazee, "Adjuvant-Enhanced Antibody Responses in the Absence of Toll-Like Receptor Signaling,” Science, Vol. 314, No. 5807, 2006, pp. 1936-1938. doi:10.1126/science.1135299

[12] M. J. Crumpton, "Protein Antigens: The Molecular Bases of Antigenicity and Immunogenicity,” In: M. Sela, Ed., The Antigens, Academic Press, New York, San Francisco, London, 1974, pp. 1-78.

[13] B. Benaceraf and E. R. Unanue, Textbook of Immunology, 1969, pp. 23-29, 54-75.

[14] F. Kurzer and K. Donraghi-Zadeh, "Advances in the Chemistry of Carbodiimides," Chemical Reviews, Vol. 67, No. 2, 1967, pp. 107-152. doi:10.1021/cr60246a001

[15] E. A. Kabat and M. M. Mayer, "Experimental Immunochemistry,” Thomas, Spring Field, 1961.

[16] H. G. Khorana, "Observations on the Use of Dicyclohexyl carbodiimide in the Synthesis of Peptides," Chemical Industries, London, 1955.

[17] S. AL-Safi and F. H. Kamel, "Efficiency of Three Me- 
thods in Separation Highly Purified Human IgG," Iraqi Journal of Chemistry, Vol. 19, No. 1, 1994, pp. 104-122.
[18] P. Delves and I. Roitt, "Encyclopedia of Immunology," 2nd Edition, Academic Press Inc., San Diego, 1999. 